\title{
CSO to Solve Optimal Demand for TOU Customers in
}

\section{Power Systems}

\author{
Wei-Nong Chen ${ }^{1}$, Zheng-Yi Chen ${ }^{2}$ and Jung-Chin Chen $^{3}$ \\ 1. Department of Industrial Engineering and Management, Cheng Shiu University, Kaohsiung 833, Taiwan \\ 2. Department of Computer Science and Information Engineering, Li-Chih Valuable School, Kaohsiung 807, Taiwan \\ 3. Department of Electronic Engineering, National Kaohsiung University of Applied Sciences, Kaohsiung 807, Taiwan
}

Received: February 11, 2015 / Accepted: April 03, 2015 / Published: July 31, 2015.

\begin{abstract}
The aim of this research is to study the optimal demand decision for the Taiwanese industries through CSO (cat swarm optimization) algorithm. The five formulations of optimal demand are developed to solve optimal contract capacity for TOU (time of use) customer. Results indicated that, the CSO algorithm is highly helpful to Taiwanese industries on the optimal demand decision. Also the CSO is superior to PSO (particle swarm optimization) in the fast convergence and better performance to find the global best solution in the same iterations.
\end{abstract}

Key words: CSO, peak loading, optimal demand, TOU, load management.

\section{Introduction}

Owing to the rapid growth of industrial and commercial, the energy sales of TPC (Taiwan Power Company) increase year by year, which results in the insufficient capacity of reserved power supply. It is therefore that, the power-rationing crisis could occur in the summer on-peak hours. This will definitely cause inconvenience for the industrial and commercial sectors and affect the civil life, and then the doubt and complaint toward the each procedure implemented by TPC. Thus, it is urgent to alleviate the power-rationing pressure through load management strategies in reducing the power demand of on-peak hours. If the study on process, equipment and power consumption characteristics of the industries with large power consumption is carried out, the load method and potentiality on power saving or transfer of on-peak hours can be created. Along with the appropriate management or electric rate incentive

Corresponding author: Jung-Chin Chen, Ph.D., research fields: optimization, fault diagnosis and load management. E-mail: chen.junchin@msa.hinet.net. package, it will efficiently reduce on-peak loading, and alleviate both power-rationing crisis and lower electricity cost.

In tandem with the draft of electric rate incentive package, the load management strategy includes the implementation of TOU (time of use) rate, the participation of interruptible load rate, the load demand control, automatic load control and the selection of optimal demand contract. Both customer and Power Company regard the rate as the production cost index. The Power Company sets up the reasonable rate structure based on the cost of supply side and the characteristics of demand side. By doing so, the rates of on-peak and off-peak hours are drawn up to reflect the power production cost in different power supply periods. While, the load management options are applied to cope with the power consumption characteristics of customer in demand side.

Optimization problems are very important in many fields. Many areas in power systems require solving one or more nonlinear optimization problems. While, analytical methods might suffer from slow 
convergence and the curse of dimensionality, heuristics-based swarm intelligence can be an efficient alternative. To the present, many optimization algorithms based on computational intelligence have been proposed [1-14], such as the GA (genetic algorithm), ACO (ant colony optimization) and PSO (particle swarm optimization) [1-5]. In this paper, a new optimization algorithm, namely, CSO (cat swarm optimization) [6] is applied to solve optimal demand decision. CSO is generated by observing the behavior of cats, and composed of two sub-models by simulating the behavior of cats. According to the experiments, the results reveal that $\mathrm{CSO}$ is superior to PSO.

In sum, it is hoped that, the proposed conclusion will be adopted by the Taiwanese industry to implement the optimal demand contract with the benefit of decreasing rate cost. Also this research aims at exploring the benefit on load management options and to provide decision-makers and leaders with useful operation and management strategies as reference.

\section{The Problem and Objective Function}

With the rapid growth of air conditioner load, the peak loading of customer in summer daytime period increases dramatically and the condition of peak loading in 15 min leading demand contract becomes more serious. According to the electric price system in TPC, customers are asked to pay extra cost with respect to the portion of basic fee in the case that the peak loading is higher than the demand contract. On the other hand, the inappropriate higher demand contract setting can avoid the occurrence of previous stated problem but will result in another problem of higher basic electric fee payment. The basic idea of optimal demand contract strategy is to derive a better demand contract such that the annual electric basic cost can be minimized [15-20]. In other words, the maximum demand is measured by $15 \mathrm{~min}$ in average. In case of customer's actual maximum demand, is in excess of the contracted capacity, the demand charge of the excess within $10 \%$ of the contracted capacity is charged twofold of the rate of contracted capacity, the excess over $10 \%$ of the contracted capacity is charged threefold of the rate of contracted capacity.

For industry customer taking TOU rate service is shown in Table 1. In Fig. 1, the regular contracted demand is determined according to the agreement between the customer and the Power Company on the basis of customer's maximum demand during peak period in summer $(A=B P$, regular contracted demand). The non-summer contracted demand is the demand in excess of the regular contracted demand during peak period in non-summer. The Saturday partial-peak period contracted demand is the demand in excess of the sum of the regular contracted demand and the non-summer contracted demand during Saturday partial-peak period $(B-A=B M$, partial-peak contracted demand). The off-peak period contracted demand is the demand in excess of the sum of the regular contracted demand, the non-summer contracted demand and the Saturday partial-peak contracted demand during off-peak period $(C-B=$ $B O$, off-peak contracted demand).

The computation of monthly electric basic fee in three conditions is expressed as follows. Based on Eq. (1), the formulation of optimal demand contract with linear programming form is obtained in Eqs. (2)-(5), respectively. In these equations, the $P_{\text {opt }}$ is within the interval of between $P_{\min }$ and $P_{\max }$. Also, the terms of $B C\left(P_{\text {opt }}\right)$ and $E C\left(P_{\text {opt }}\right)$ mean the basic electric fee and extra payment. It is noted that, the $P_{\text {opt }}$ affects both these two terms.

The formulation of optimal demand contract is expressed as follows:

$$
\min T C=\sum_{i=1}^{12}\left[B C_{i}(B P, B M, B O)+E C_{i}\left(P P_{i}, P M_{i}, P O_{i}\right)\right]
$$

where, $T C$ : total electric basic cost in a year; $B C$ : total electric basic cost in a year; $B C_{i}(B P, B M, B O)$ : ith month electric basic fee; $B P$ : peak loading demand contract engaged with TPC $(\mathrm{kW}) ; B M$ : partial-peak 
Table 1 The extra high tension customer.

\begin{tabular}{lllll}
\hline Classification & & & $\begin{array}{l}\text { Summer } \\
\text { (Jun. 01-Sep. 30) }\end{array}$ & $\begin{array}{l}\text { Non-summer } \\
\text { (all other days of the year) }\end{array}$ \\
\hline \multirow{3}{*}{ TOU rate } & Regular contracted demand & Per & 217.30 & 160.60 \\
& Regular contracted demand & $\mathrm{kW}$ & - & 160.60 \\
& Regular contracted demand & Per & 43.40 & 32.10 \\
& Regular contracted demand & Month & 43.40 & 32.10 \\
\hline
\end{tabular}

Source: Taiwan Power Company rate schedules.

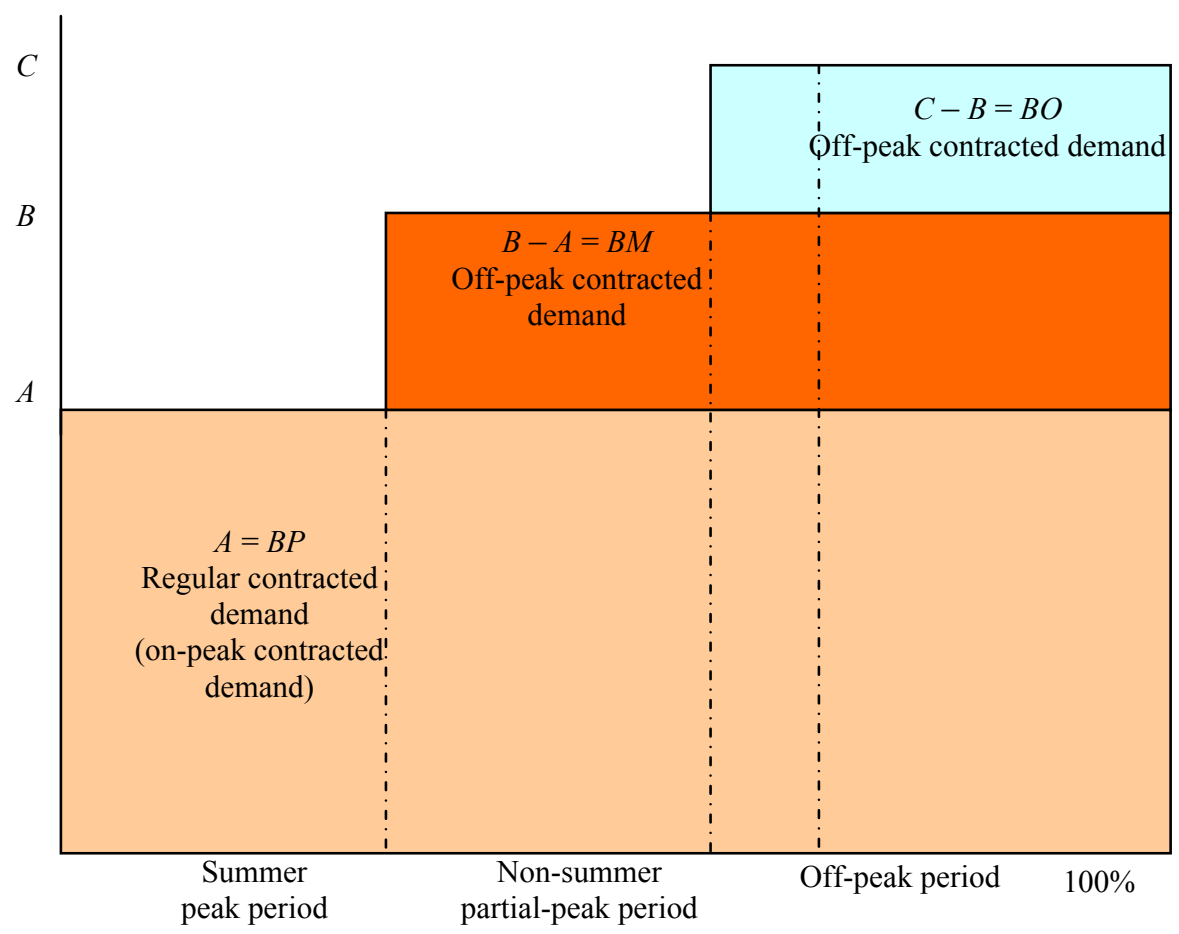

Fig. 1 The schematic diagram of optimal demand selection.

demand contract engaged with TPC $(\mathrm{kW}) ; B O$ : off-peak loading demand contract engaged with TPC $(\mathrm{kW}) ; E C_{i}$ : extra payment; $P P_{i}$ : ith month peak loading $(\mathrm{kW}) ; P M_{i}$ : ith month partial-peak loading demand; $P O_{i}$ : ith month off-peak loading demand.
The calculation of June, July, August and September (summer season) electric basic fee and extra payment are shown as follows:

$$
\begin{gathered}
B C_{i}=C P_{i} \times B P- \\
{\left[C M_{i} \times(B P+B M)\right] \times 0.5+C O_{i} \times B O}
\end{gathered}
$$

where, $E O_{i}$ : excess off-peak demand contract; $C P$ : peak demand contract fee per $\mathrm{kW}$, coefficient in summer is NT\$217.3/kW; CM: partial-peak demand contract fee per $\mathrm{kW}$, coefficient in summer is NT\$ (New Taiwan dollar)43.4/kW; $C O$ : off-peak demand contract fee per $\mathrm{kW}$, coefficient in summer is NT $\$ 43.4 / \mathrm{kW}$. 
The calculation of January, February, March, April, May, October, November and December (non-summer season) electric basic fees and extra payment are shown as follows:

$$
B C_{i}=C P_{i} \times(B P+B M)-
$$$$
\left[C M_{i} \times(B P+B M)\right] \times 0.5+C O_{i} \times B O
$$

where, $E O_{i}$ : excess off-peak demand contract; $C P$ : peak demand contract fee per $\mathrm{kW}$, coefficient in summer is NT $\$ 160.6 / \mathrm{kW}$; $C M$ : partial-peak demand contract fee per $\mathrm{kW}$, coefficient in summer is NT\$32.1/kW; CO: off-peak demand contract fee per $\mathrm{kW}$, coefficient in summer is NT $\$ 32.1 / \mathrm{kW}$.

\section{CSO to Solve Optimal Demand Contract}

This section is conducted by CSO algorithm to analyze optimal contract capacity and drop the electricity cost for Taiwanese industries. The process of CSO is described below and shown in Fig. 2:

Step (1): Create $N=5(\mathrm{Pmin}<\mathrm{P} 1, \mathrm{P} 2, \mathrm{P} 3, \mathrm{P} 4$ and P5 $<$ Pmax) in the process;

Step (2): Randomly sprinkle the cats into the M-dimensional solution space and randomly give values, which are in-range of the maximum velocity, to the velocities of every cat. Then haphazardly pick number of cats and set them into tracing mode according to mixture ratio $(M R=20 \%)$, and the others (80\%) set into seeking mode;

Step (3): Evaluate the fitness value of each cat by applying the positions of cats into the fitness function, which represents the criteria of our goal, and keep the best cat into memory. Note that we only need to remember the position of the best cat (Pbest) due to it represents the best solution so far;

Step (4): Move the cats according to their flags, if $\mathrm{cat}_{k}$ is in seeking mode, apply the cat to the seeking mode process, and otherwise apply it to the tracing mode process. The process steps are presented above;

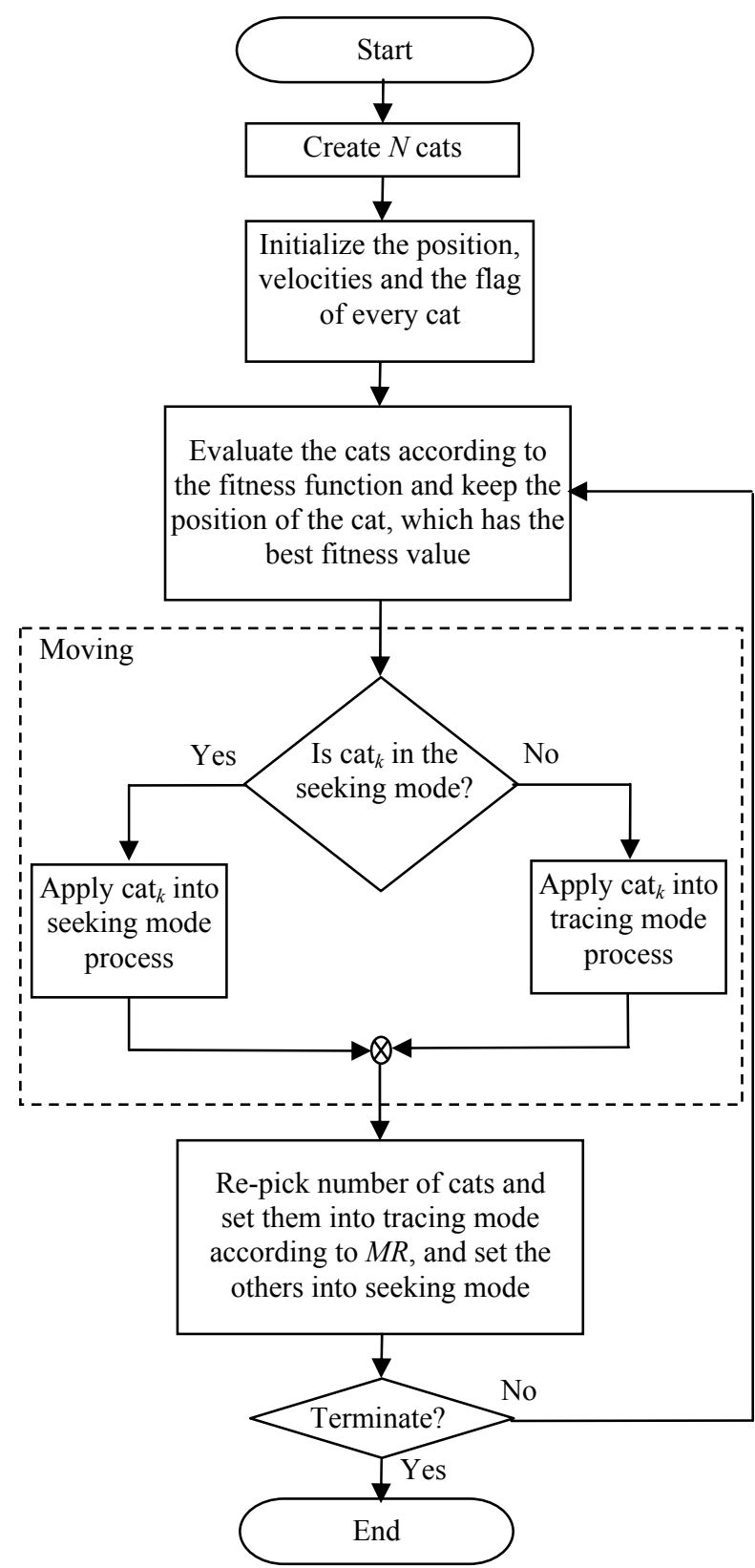

Fig. 2 The CSO research flow chat. 
Step (5): Re-pick number of cats and set them into tracing mode according to $M R$, then set the other cats into seeking mode;

Step (6): Check the termination condition, if satisfied, terminate the program, and otherwise repeat Steps (3)-(5).

\section{Experimental Results and Discussion}

\subsection{The Analysis of Power Consumption} Characteristics on Sampling Customer

This research is conducted by CSO algorithm to analyze optimal contract capacity and drop the electricity cost. In the plant visit, the current regular demand contract $(B P=29,150 \mathrm{~kW}, B M=200 \mathrm{~kW}$, $B O=150 \mathrm{~kW})$ is not well implemented for customer A. As the customer A roughly estimates the optimal demand contract based on the historical information and does not rigidly analysis by the mathematical method. Thus, the expenditure of demand charges increase. After explanation and analysis, the customer willingly precedes the implementation of optimal contract capacity. The analysis of power consumption characteristics shows in Table 2.

Table 2 The analysis of power consumption characteristics on customer $A$.

\begin{tabular}{|c|c|c|c|}
\hline \multicolumn{4}{|c|}{$B P=29,150 \mathrm{~kW}, B M=200 \mathrm{~kW}, B O=150 \mathrm{~kW}$} \\
\hline Monthly & PPi $(\mathrm{kW})$ & PMi (kW) & POi $(\mathrm{kW})$ \\
\hline 1 & 0 & 30,312 & 30,451 \\
\hline 2 & 0 & 30,009 & 30,147 \\
\hline 3 & 0 & 29,527 & 29,600 \\
\hline 4 & 0 & 29,654 & 29,726 \\
\hline 5 & 0 & 29,774 & 29,847 \\
\hline 6 & 29,316 & 29,986 & 30,059 \\
\hline 7 & 29,374 & 29,506 & 29,736 \\
\hline 8 & 28,995 & 28,224 & 29,683 \\
\hline 9 & 29,141 & 29,683 & 29,832 \\
\hline 10 & 0 & 29,847 & 29,526 \\
\hline 11 & 0 & 29,688 & 29,369 \\
\hline 12 & 0 & 29,482 & 29,615 \\
\hline
\end{tabular}

PPi: customer monthly peak loading $(\mathrm{kW})$; PMi: customer monthly partial -peak loading $(\mathrm{kW})$; POi: customer monthly off-peak loading $(\mathrm{kW}) ; T C=\mathrm{NT} \$ 64,740,836$.

\subsection{Parameter Settings for CSO and PSO}

For CSO, we set the parameters, which have been discussed in the above-mentioned according to Table 3. The parameter settings for PSO and PSO parameters are listed in Table 4.

\subsection{Experimental Results for CSO and PSO}

In the experiments for all test function, we aim at finding the minimum of the fitness value, in other words, the goal of our experiments is to minimize the electricity cost. For CSO and PSO, we apply 50 iterations per cycle to compare the performance and convergence condition. We found that, $\mathrm{CSO}$ is earlier convergence than PSO in iteration number.

The results of test optimal demand contract and the convergence curves are shown in Figs. 3-6, respectively. The horizontal axis represents the iterations, and the vertical axis represents the fitness values (minimize cost).

Figs. 3-6 show the convergence curve for CSO and PSO on optimal demand contract with 50 iterations, also we have the best value of $B P=29,264 \mathrm{~kW}, B M=$ $487 \mathrm{~kW}, B O=346 \mathrm{~kW}$. CSO showed significant fast convergence about four iterations (minimum cost is NT\$64,032,044). The results indicated that CSO

Table 3 Parameter settings for CSO.

\begin{tabular}{ll}
\hline Parameter & Value or range \\
\hline SMP (seeking memory pool) & 5 \\
SRD (seeking range of the dimension) & $30 \%$ \\
CDC (counts of dimension to change) & $100 \%$ \\
$M R$ & $20 \%$ \\
$c_{1}$ & 2.0 \\
$r_{1}$ & {$[0,1]$} \\
\hline
\end{tabular}

The $c_{1}$ is a constant and $r_{1}$ is a random value in the range of $[0,1]$.

Table 4 Parameter settings for PSO.

\begin{tabular}{ll}
\hline Parameter & Value or range \\
\hline$c_{1}$ & 2.0 \\
$c_{2}$ & 2.0 \\
$r_{1}$ & {$[0,1]$} \\
$r_{2}$ & {$[0,1]$} \\
\hline The $c_{1}$ and $c_{2}$ is a constant. We set $c_{1}=c_{2}=2, r_{1}=r_{2}$ a random
\end{tabular}
value in the range of $[-1,1]$ 
presents a better performance of finding the global best solution.

Table 2 indicates the monthly peak load for the sampled customer and the peak load in the scope of month is within $28,995-29,374 \mathrm{~kW}$, partial-peak load within 28,224-30,312 kW, off-peak load within 29,369-30,451 kW. This sampling customer current regular demand contract capacity is $2,915 \mathrm{~kW}(B P)$,
$200 \mathrm{~kW}(B M)$, and $150 \mathrm{~kW}(B O)$. To sum up, the sampling customer may adjust the contract capacity and can save a considerable electricity rate. If this customer selects optimal contract capacity, it will save (NT\$64,740,836 - NT\$64,032,044) NT\$708,792 annual rate.

Due to the characteristics of process and equipment running, the sampling customer can further implement

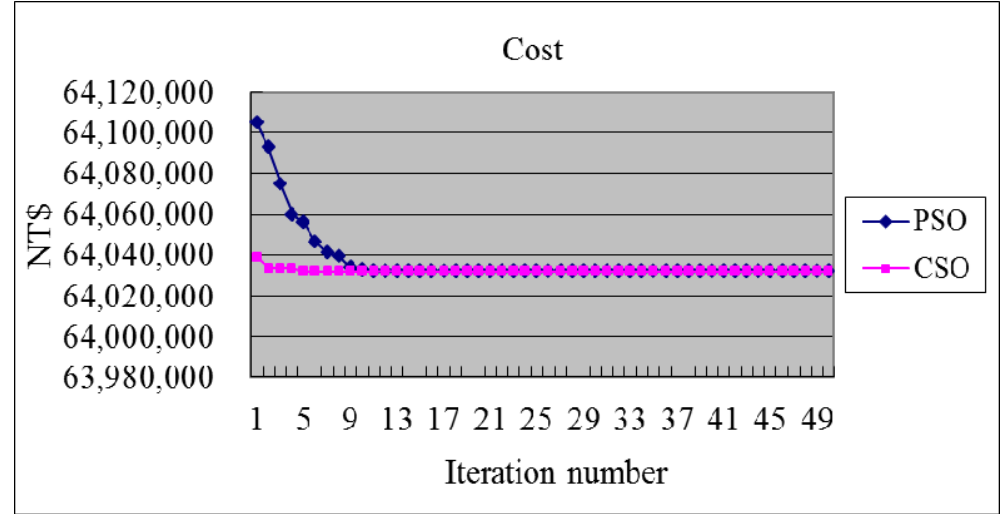

Fig. 3 The convergence curve of CSO and PSO in cost.

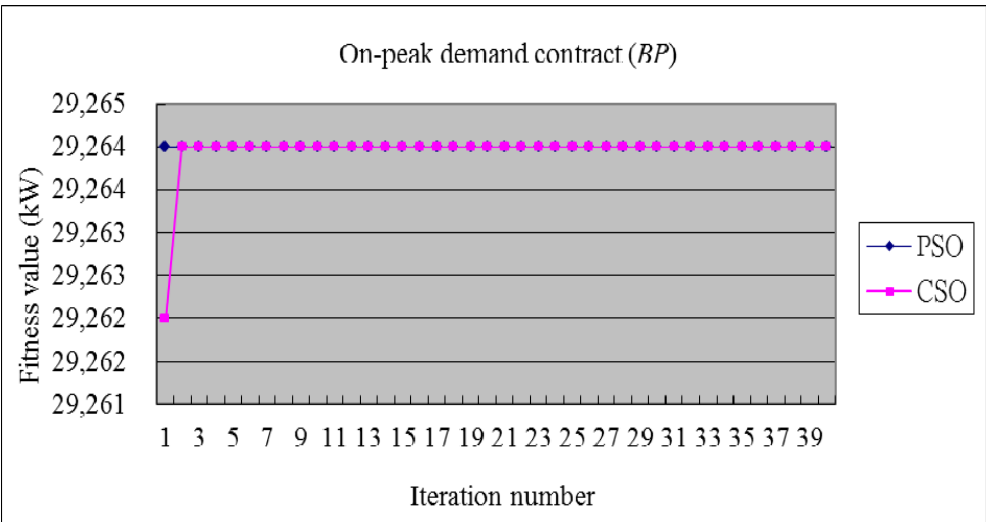

Fig. 4 The convergence curve of CSO and PSO in $B P$.

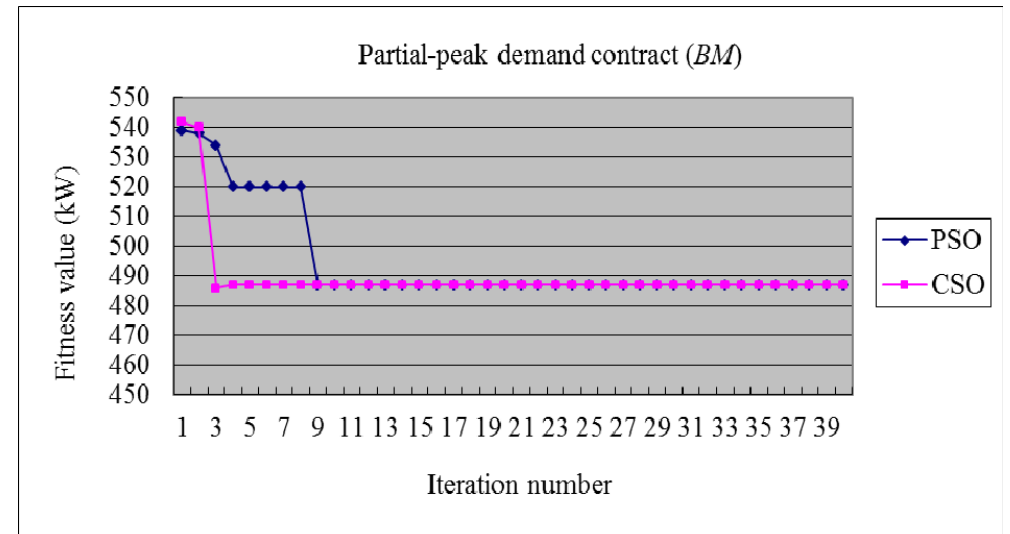

Fig. 5 The convergence curve of CSO and PSO in BM. 


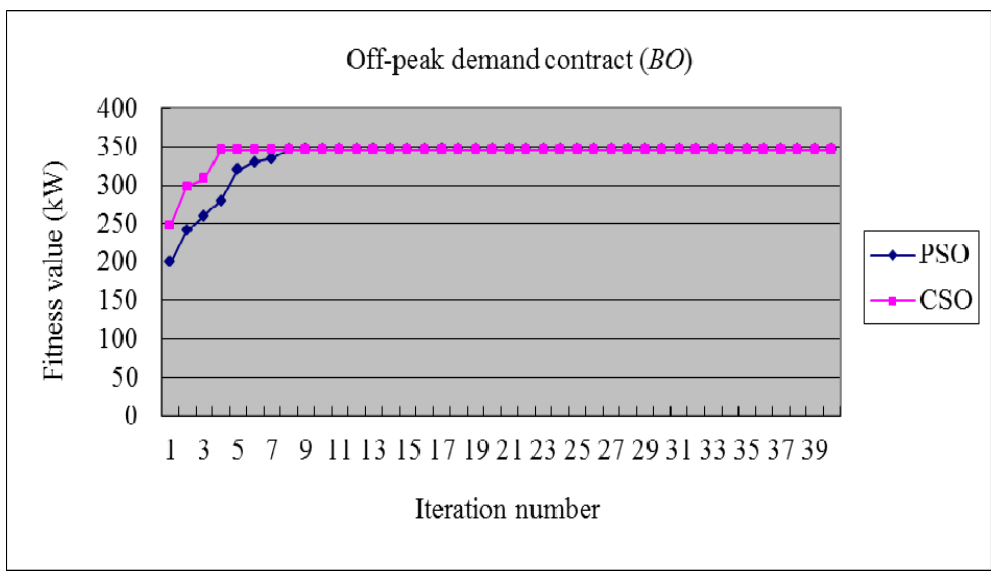

Fig. 6 The convergence curve of CSO and PSO in $\mathrm{BO}$.

the TOU rate options. The customers expressed their interests in the daily load curve variation of the power consumption. In line with the power consumption and rate analytical variation of on-peak \& off-peak hours provided by this research, the customer realizes that, the TOU rate measures will save its electricity cost in very short time. Thus the customer participates in the load management options. Meanwhile, the customer will consider how to change process to cope with various beneficial rate options provided by TPC.

CSO showed significant faster convergence about 7th iterations, and PSO showed significant convergence after 11th iterations. In other word, CSO is faster convergence than PSO on optimal demand contract selection.

The Visual Basic 6.0 language and Microsoft Excel 97 were adopted as the developing tool to carry out the proposed work. Also executing 50 times CSO and PSO program, the CSO mean squared deviation $(\mathrm{MAD}=0.0273 \%)$ is lower error than the PSO (MAD $=0.2438 \%$ ). The results indicated that, CSO presents a better performance of finding the global best solution than PSO.

\section{Conclusions and Suggestions}

This paper presents a brief literature review of the optimal demand contract and proposes five formulations of importance to TOU customer. These formulations are developed as a framework which could serve to help industry customer implementing optimal demand policy, and CSO can be effectively applied to solve optimal contract capacity.

From the results, CSO presents a better performance of finding the global best solution. Though CSO takes more time to finish the same iteration than PSO algorithms, it improves the performance of finding global set solutions. But if considering the same iteration time, CSO still presents a higher performance than PSO algorithms. The results indicated that, $\mathrm{CSO}$ is faster convergence than PSO on optimal demand contract selection. Also, the CSO can be effectively applied to different optimization problems in power systems. It is helpful to implement the optimal demand contract, load management and to drop electricity cost for TOU industry customers. It is suggested that, the Taiwanese industries should review the ratio between current capacity and demand contract in order to decrease the demand contract or participate in the interruptible rate package for the reduction of demand charge.

\section{Acknowledgments}

The author would like to express my gratitude to my supervisor Dr. Pan and Dr. Hwang, for my advice and suggestions, without which much of this work would have been impossible.

\section{References}

[1] Kim, K. W., Gen, M., and Kim, M. H. 2006. "Adaptive Genetic Algorithms for Multi-resource Constrained Project Scheduling Problem with Multiple Modes." 
International Journal of Innovative Computing, Information \& Control 2 (1): 41-9.

[2] Maeda, Y., and Li, Q. 2005. "Parallel Genetic Algorithm with Adaptive Genetic Parameters Tuned by Fuzzy Reasoning." International Journal of Innovative Computing, Information \& Control 1 (1): 95-107.

[3] Chu, S. C., Roddick, J. F., and Pan, J. S. 2004. "Ant Colony System with Communication Strategies." Information Sciences 167 (1-4): 63-76.

[4] Kennedy, J., and Eberhart, R. 1995. "Particle Swarm Optimization." In Proceedings of the IEEE International Conference on Neural Networks, 601-10.

[5] Chu, S. C., Roddick, J. F., Su, C. J., and Pan, J. S. 2004. "Constrained ant Colony Optimization for Data Clustering." In Proceedings of the 8th Pacific Rim International Conference on Artificial Intelligence, 534-43.

[6] Chu, S. C., Tsai, P. W., and Pan, J. S. 2006. "Cat Swarm Optimization." In Proceedings of the 9th Pacific Rim International Conference on Artificial Intelligence, 854-8.

[7] Dokopoulos, P. S., Saramourtsis, A. C., and Bakirtzis, A. G. 1996. "Prediction and Evaluation of the Performance of Wind-Diesel Energy Systems." IEEE Trans. on Energy Conversion 11 (2): 385-93.

[8] Karaki, S. H., Chedid, R. B., and Ramadan, R. 2000. "Probabilistic Production Costing of Diesel-Wind Energy Conversion Systems." IEEE Trans. on Energy Conversion 15 (3): 284-9.

[9] Chen, C. L., and Chen, N. 2001. "Multi-area Economic Generation and Reserve Dispatch." In Proceedings of the IEEE PICA (Power Industry Computer Application) Conference, 368-73.

[10] Walters, D. C., and Sheble, G. B. 1993. "Genetic Algorithm Solution of Economic Dispatch with Valve Point Loading." IEEE Trans. on Power Systems 8 (3): 1325-31.

[11] Lin, W. M., Cheng, F. S., and Tsay, M. T. 2002. "An
Improved Tabu Search for Economic Dispatch with Multiple Minima." IEEE Trans. on Power Systems 17 (1): 108-12.

[12] Yang, H. T., Yang, P. C., and Huang, C. L. 1996. "Evolutionary Programming Based Economic Dispatch for Units with Non-smooth Fuel Cost Functions." IEEE Trans. on Power Systems 11 (1): 112-8.

[13] Gaing, Z. L. 2003. "Particle Swarm Optimization to Solving the Economic Dispatch Considering the Generator Constraints." IEEE Trans. on Power Systems 18 (3): 1187-9.

[14] Chen, C. L., and Chen, N. 2001. "Direct Search Method for Solving Economic Dispatch Problem Considering Transmission Capacity Constraints." IEEE Transaction on Power Systems 16 (4): 764-9.

[15] Hsiao, H. C., Hsiao, C. Y., Lin, M. C., Wu, Q. F., and Chan, T. C. 1990. "Forecasting the Optimal Contract Capacities in Industrial Distribution Systems." In Proceedings of the 11th Symposium on Electrical Power Engineering, 215-20.

[16] Yang, C. G., Shu, R. T., and Yang, G. K. 1991. Load Management, Determination for the Optimal Contract Capacities. Load Management Part II, Taiwan Power Company Internal report.

[17] Lee, T. Y., and Chen, N. 1994. "Optimal Utility Contracts for Time-of-Use Rates Industrial Customers." Journal of the Chinese Institute of Electrical Engineering 1 (4): 247-57.

[18] Cohen, A. I. 1988. "An Optimization Method for Load Management Scheduling." IEEE Trans. on Power Systems 3 (2): 612-8.

[19] Chen, J., Lee, F. N., Breiphl, A. M., and Adapa, R. 1995. "Scheduling Direct Load Control to Minimize System Operation Cost." IEEE Trans. on Power Systems 10 (4): 1994-2000.

[20] Wei, D. C., and Chen, N. 1995. "Air Conditioner Direct Load Control by Multi-pass Dynamic Programming." IEEE Trans. on Power Systems 10 (1): 307-13. 